\title{
Active Atoms and Interstitials in Two-Dimensional Colloidal Crystals
}

\author{
Kilian Dietrich, ${ }^{1}$ Giovanni Volpe, ${ }^{2}$ Muhammad Nasruddin Sulaiman, ${ }^{3}$ Damian Renggli, ${ }^{1}$ Ivo Buttinoni,,${ }^{1,3,}$ and Lucio Isa ${ }^{1, \dagger}$ \\ ${ }^{1}$ Laboratory for Interfaces, Soft Matter and Assembly, Department of Materials, ETH Zurich, Zurich 8093, Switzerland \\ ${ }^{2}$ Department of Physics, University of Gothenburg, Göteborg 41296, Sweden \\ ${ }^{3}$ Physical and Theoretical Chemistry Laboratory, Department of Chemistry, Oxford OX1 3QZ, United Kingdom
}

(Received 11 October 2017; revised manuscript received 29 May 2018; published 29 June 2018)

\begin{abstract}
We study experimentally and numerically the motion of a self-phoretic active particle in two-dimensional loosely packed colloidal crystals at fluid interfaces. Two scenarios emerge depending on the interactions between the active particle and the lattice: the active particle either navigates throughout the crystal as an interstitial or is part of the lattice and behaves as an active atom. Active interstitials undergo a run-and-tumblelike motion, with the passive colloids of the crystal acting as tumbling sites. Instead, active atoms exhibit an intermittent motion, stemming from the interplay between the periodic potential landscape of the passive crystal and the particle's self-propulsion. Our results constitute the first step towards the realization of nonclose-packed crystalline phases with internal activity.
\end{abstract}

DOI: 10.1103/PhysRevLett.120.268004

Synthetic active particles, including phoretic microswimmers powered by local chemical [1], thermal [2], and ionic gradients [3], interact with obstacles differently than Brownian particles [4]. Pillars (or large passive spheres) can, for example, steer or trap active particles as a result of short-range interactions [5-7]. Self-propelling particles confined in semidilute colloidal suspensions have also unveiled a wealth of new physics: active colloids can exert an effective pressure $[8,9]$ that promotes local compression and melting of clusters [10] as well as the segregation of active and passive species [11]. Understanding how self-propelled constituents affect even denser structures, e.g., crystals or glasses, may pave the way for the fabrication of new phases of matter. Recent numerical simulations showed, for instance, that the presence of excited particles drastically alters the topological features in crystalline structures [12,13] and introduces novel dynamic properties in arrested glassy phases [14,15]. Despite this growing interest, experiments aimed at studying the motion of active particles in dense crystals or glasses are lagging significantly behind simulations, mainly because single-particle activity is strongly weakened in closed-packed active suspensions, where neighboring colloids screen the phoretic gradients responsible for selfpropulsion [16-18]. Consequently, experimental studies of active crystals and glasses have been so far limited to dry granular suspensions of macroscopic particles [19].

Here, we study the motion of active Janus colloidal particles within loosely packed crystalline monolayers formed by spreading passive (Brownian) charged colloids at a flat water-oil interface [20], which enables us to avoid the reduction of activity happening in close-packed samples. In fact, long-ranged repulsive electrostatic dipolar forces at the interface [21] give rise to 2D crystalline arrays with lattice spacings significantly larger than the particle diameter. Self-propelling Janus colloids show a varying degree of electrostatic coupling to these lattices, and behave either as active interstitials or as active atoms. For weak coupling, active interstitials can be viewed as self-propelling particles navigating within an array of spherical obstacles, i.e., the passive colloids. Although the full microscopic details of the interactions at short range are still to be disclosed, the obstacles can reorient the active trajectories in a run-and-tumble-like fashion. For large coupling, active atoms are effectively part of the crystal and self-propel while keeping a large distance (of the order of the lattice constant) from their neighbors. These types of particles are promising building blocks for the fabrication and study of $2 \mathrm{D}$ active crystals and glasses. These two scenarios depend on the orientation of the Janus colloids relative to the interface [22], irrespective of the magnitude of the swimming velocity.

We perform our experiments at a flat water-hexadecane interface [23]. Fluorescent polystyrene particles (PS, Microparticles $\mathrm{GmbH}$ ) with a radius of $R=1.4 \mu \mathrm{m}$ are placed at the interface via injection of a $1: 1$ waterisopropyl alcohol spreading solution [22]. A small amount $(\approx 1 \%)$ of the particles have one hemisphere coated by sputtering with a $2 \mathrm{~nm}$ layer of $\mathrm{Pt}$, which acts as a catalyst and triggers the decomposition of $\mathrm{H}_{2} \mathrm{O}_{2}$ in the aqueous phase, thus leading to active motion where both translation and rotation occur within the two-dimensional (2D) $x y$ plane of the interface $[22,30]$. The use of nonstabilized $\mathrm{H}_{2} \mathrm{O}_{2}$ and the roughness of the sputtered coatings promote swimming velocities that are approximately one order of magnitude larger than the values reported in the literature for catalytic microswimmers (values are compared for colloids swimming on a solid substrate [23]). After spreading, passive PS particles self-assemble at the interface into 
loosely packed crystalline monolayers (Video S1 [23]) due to dipolar, pair-additive, repulsive forces with a potential $U_{p p} /\left(k_{B} T\right)=a_{p p} r^{-3}$, which stems from asymmetric charge effects across the interface [20,31]. The lattice constant $L$ is determined by the amount of spread particles. At a given center-to-center distance $r$, the dipole magnitude depends on the surface charge and the contact angle $\left(\theta_{p}=123^{\circ} \pm 8^{\circ}[22]\right)$ of the passive particle pairs [31].

Upon exposure to the $\mathrm{H}_{2} \mathrm{O}_{2}$-rich aqueous phase, the Pt-coated PS particles swim with a velocity $V_{0}$ (free swimming velocity, i.e., in the absence of the lattice), which, for a given $\mathrm{H}_{2} \mathrm{O}_{2}$ concentration, crucially depends on the orientation of the Pt-coated hemisphere with respect to the interface plane, as we have shown in our previous work [22]. Two predominant configurations, distinguishable by the particle brightness in fluorescence microscopy, are found: particles either have the Pt cap mostly wetted by the oil phase [active interstitials, Fig. 1(a)] or by the water phase [active atoms, Fig. 1(b)] [22]. The former configuration is typically 5-10 times faster than the latter [22]. In the crystal, these active particles show similar contact angles $\left(\theta_{a}=104^{\circ} \pm 10^{\circ}\right.$ [22] $)$ as the passive ones, but, because of the uncharged Pt cap, their orientation strongly affects the interactions with the surrounding passive colloids. The dipolar interaction with the particles in the lattice will therefore have a form $U_{p a}=c U_{p p}$, where $c$ is an orientation-dependent coupling parameter. Active interstitials [Fig. 1(a)] are characterized by a weak coupling and navigate through the crystal [blue trajectory in Fig. 1(c) and Video S2 [23] ] without perturbing it, as shown by the trajectories of the neighboring passive colloids (black). Instead, active atoms [Fig. 1(b)] interact strongly with the crystal and their active motion [red trajectory in Fig. 1(d) and Video S3 [23] ] leads to a localized structural response of the monolayer [black trajectories in Fig. 1(d)].

Our understanding of the experiments is verified by a minimalistic numerical model based on Brownian dynamics simulations of the passive and active microspheres $(R=1.4 \mu \mathrm{m})[23,32]$. The amplitude of the dipolar interactions $a_{p p}=5 \times 10^{-13} \mathrm{~m}^{3}$ is chosen in agreement with previous literature [21] and a short-range effective torque is added to reorient the active particle when the center-tocenter distance with a passive is smaller than $4 R[23,33]$ (see discussion in the following paragraph). After equilibration of the lattice, we provide one microparticle with a coupling $c$ and free swimming velocity, which is chosen to match the experimental $V_{0}$, measured in [22]. Simulations at low coupling [ $c=0.001$, Fig. 1(e) and Video S4 [23] ] reproduce the dynamics of active interstitials, whereas simulations at high coupling $[c=1$, Fig. 1(f) and Video S5 [23] ] exhibit features that are consistent with the dynamics of active atoms [Fig. 1(b)]. Quantitative agreement is also found for the velocity [Fig $1(\mathrm{~g})]$ and nearest-neighbor distance distributions [Fig. 1(h)], for

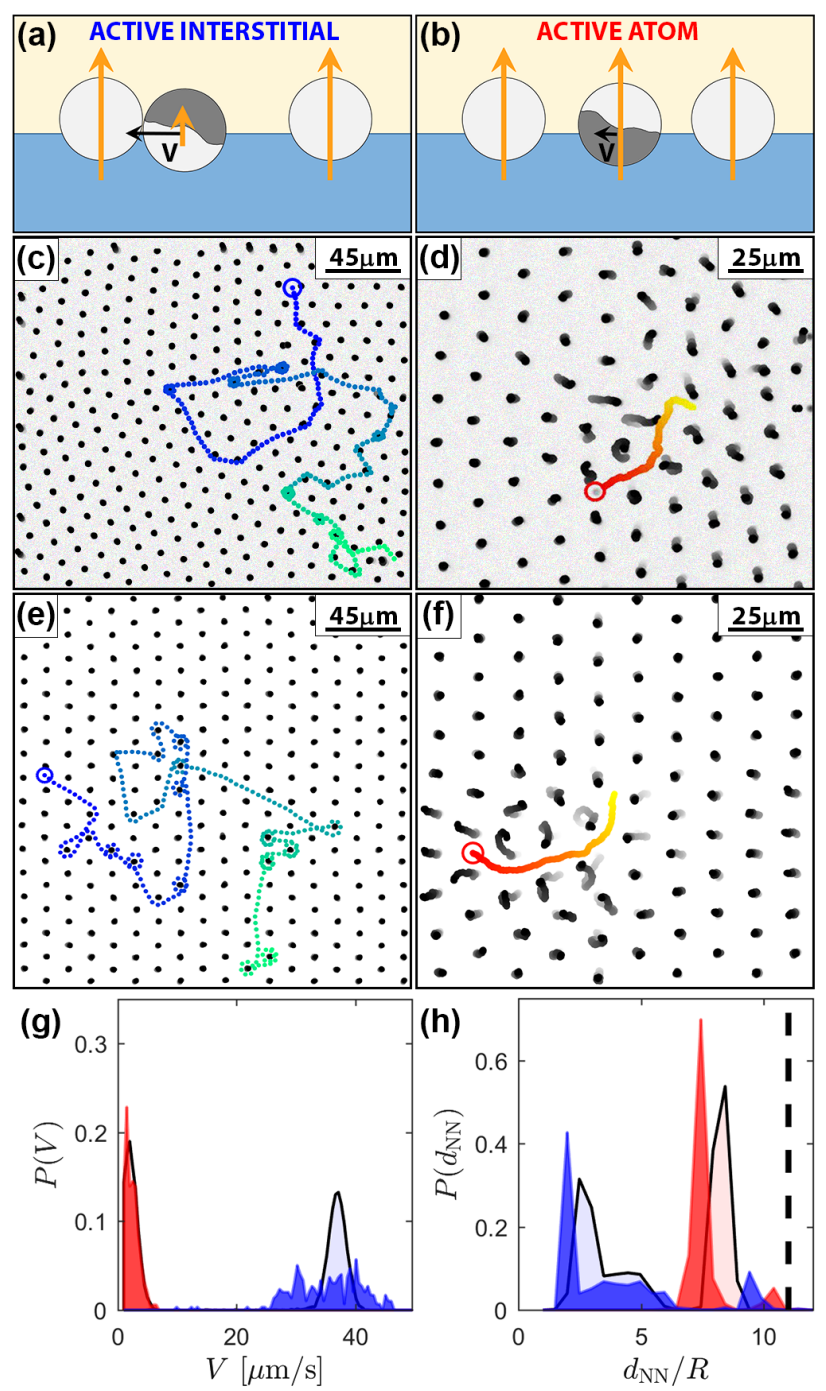

FIG. 1. Active particles in a loosely packed colloidal crystal with lattice constant $L$. (a),(b) Interactions between active and passive particles at the water-oil interface: (a) active interstitial and (b) active atom. The orange arrows sketch the magnitude of the dipolar coupling. (c),(d) Experimental trajectories and (e),(f) corresponding numerical simulations. Blue, active interstitial. Red, active atom. Black, passive colloids. The color gradients represent time from $t=0 \mathrm{~s}$ (green-yellow-gray) to $t=30 \mathrm{~s}$ (blue-red-black). $L=10 R, V_{0}=38 \mu \mathrm{m} / \mathrm{s}$ (active interstitials, coupling $c=0.001$ ) and $V_{0}=6 \mu \mathrm{m} / \mathrm{s}$ (active atoms, coupling $c=1$ ). (g) Velocity and (h) normalized nearest-neighbor distance (center-to-center) probability distributions for the trajectories shown in (c), (d), (e), and (f). Solid histograms, experiments. Transparent histograms, simulations. The dashed line in (h) marks the experimental lattice constant. Experiments are made at $\left[\mathrm{H}_{2} \mathrm{O}_{2}\right]=3 \%$ and images are recorded at $10 \mathrm{fps}$.

which direct contact is only achieved at low coupling [blue data in Fig. 1(h)].

We first focus on the behavior of the active interstitials. Figures 2(a)-2(f) show three experimental [Figs. 2(a)-2(c), Videos S6-S8 [23]] and numerical [Figs. 2(d)-2(f)] trajectories of active interstitials inside $2 \mathrm{D}$ crystals with 


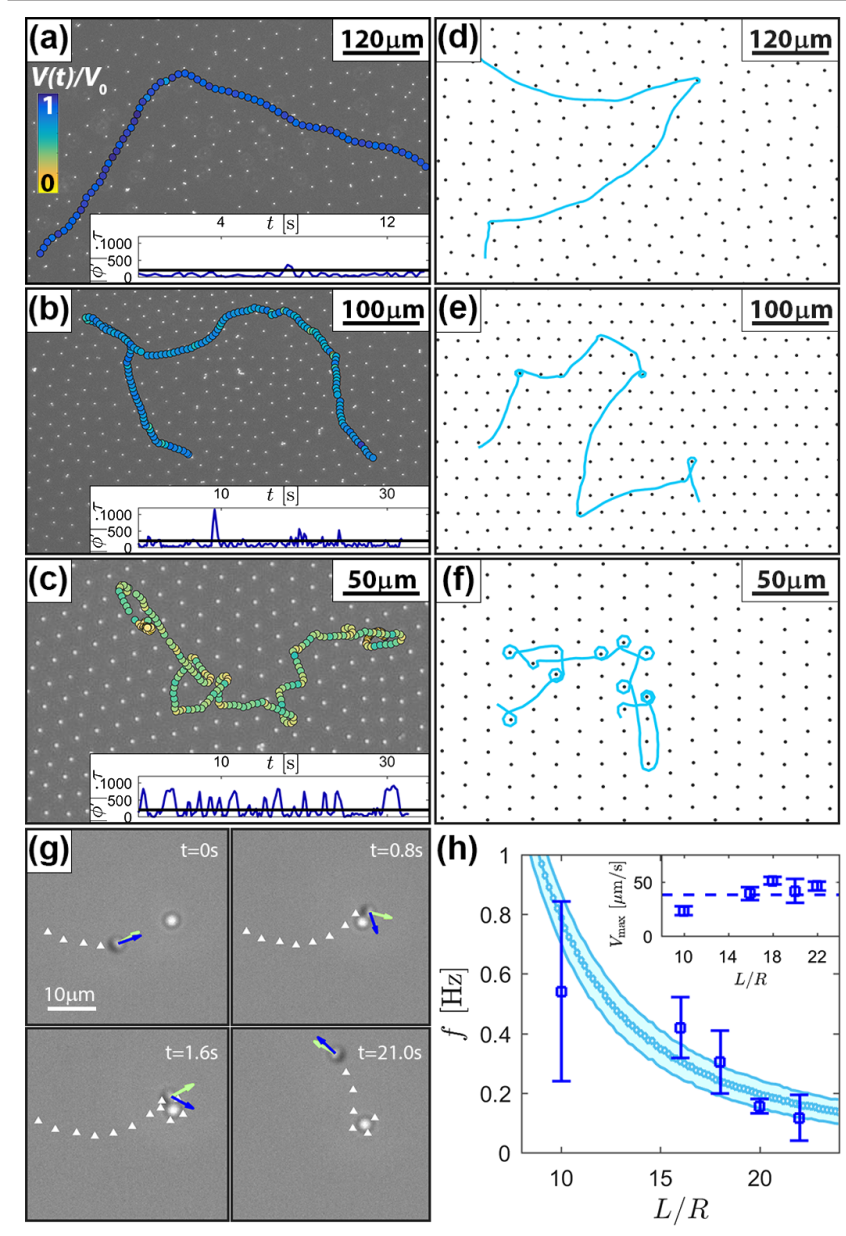

FIG. 2. Active interstitials. (a)-(c) Experimental trajectories of active interstitials in colloidal lattices with (a) $L=22 R$, (b) $L=16 R$, and (c) $L=10 R\left(\left[\mathrm{H}_{2} \mathrm{O}_{2}\right]=3 \%, V_{0}=38 \mu \mathrm{m} / \mathrm{s}\right)$. The insets show the experimental absolute angular velocity $\left|\phi^{\prime}\right|$ measured in sequential frames. When $\left|\phi^{\prime}\right|$ reaches a value greater than $200 \tau^{-1}$ (horizontal black line, $\tau$ is the free rotational diffusion time), a tumbling event is registered (see also [23]). The color code highlights the relative particle speed. (d)-(f) Corresponding numerical simulations obtained using $c=0.001$ and $V_{0}=38 \mu \mathrm{m} / \mathrm{s}$. (g) Frame sequence of an active interstitial interacting with a passive colloid. The direction of the swimming velocity (blue arrow) with respect to the particle orientation (green arrow) changes when the swimmer is captured. Details of the detection of the particle orientation are in [23]. (h) Experimental (blue) and numerical (cyan) tumbling frequency plotted as a function of the lattice constant. The inset shows that the maximum speed corresponds to the free swimming velocity $V_{0}=38 \mu \mathrm{m} / \mathrm{s}$ (dashed horizontal line).

decreasing lattice constant $(L=22 R, 16 R$, and 10R). In all cases, the passive monolayer acts as a pinball machine: the active interstitial swims along straight paths with large persistence $[22,30]$ and reorients abruptly when it is in the proximity of a passive colloid by orbiting around it. In fact, when the microswimmer approaches a passive particle [Fig. 2(g) and Video S9 [23] ], its direction of motion (blue arrow) changes from that observed before the collision and no longer corresponds to the $2 \mathrm{D}$ projection of the particle asymmetry [green arrow, note that in $2(\mathrm{~g})$ a $20 \mathrm{~nm}$ Pt layer is used to visualize the cap and this choice leads to a metastable tilted orientation [23,34]]. Eventually, the microswimmer escapes the passive obstacle in a random direction [23]. This scattering event randomizes the activemotion direction by enforcing a reorientation of the velocity vector. The orbiting of active particles around large circular obstacles has been observed in other recent works [5-7] and has been ascribed to hydrodynamic coupling or to local deformations of the chemical gradient, although other interactions (e.g., electrostatic and van der Waals) cannot be fully neglected. In our system, there is another possible source of transient trapping. Because of contact line undulations, two particles confined at a fluidfluid interface experience reciprocal capillary attractions whose magnitude depends on their contact angle and surface roughness and decays as $r^{-4}$ [35], thus steeper than the dipolar repulsion (at short distances, additional terms in the electrostatic interactions may be present [36], but do not alter the interpretation of the data). Assuming a surface roughness of the colloids of the order of few $\mathrm{nm}$ and a coupling $c=0.001$, the resulting potential (sum of electrostatic repulsions and capillary attractions) close to contact is $\sim 50 k_{B} T$ [23]. These values fall within the range of effective temperatures [37] of active colloids reported by Ginot et al. [8]; i.e., the self-propulsion is sufficiently strong to allow the particles to escape contact, even more so for the larger velocities considered in our work. In our simulations, we account for the orbiting dynamics by phenomenologically introducing an effective torque $[23,33]$, which is only present when the active interstitial is at distances $<4 R$ from a passive sphere (additional theoretical work is needed to clarify the microscopic physics that produce this torque). The resulting motion of the active interstitial is determined by the probability of collisions with a passive colloid, as defined by the lattice constant of the crystal [Fig. 2(h)]. At fixed $\left[\mathrm{H}_{2} \mathrm{O}_{2}\right]$, the swimming velocity, evaluated as the maximum speed in the lattice [inset to 2(h)], remains constant and equal to the propulsion velocity measured at pristine interfaces (dashed line). A small decrease is observed only for the densest crystal $(L=10 R)$.

Active atoms swim at distances from the passive particles that are significantly larger than $4 R$ [Fig. 1(h), red data] and do not experience local trapping around the passive spheres. Because of strong coupling, active atoms instead feel the local electrostatic potential landscape of the two-dimensional crystal and exhibit large velocity fluctuations as they explore several unit cells [Fig. 3(a)]. In particular, events when the active atom swaps cells are marked by the vertical lines and correspond to the velocity peaks. Figures 3(b)-3(d) show the Voronoi tessellation of an image sequence taken across one of the peaks in Fig. 3(a). The velocity of the active atom, proportional to the length of the red arrow, increases abruptly [Fig. 3(c) and 

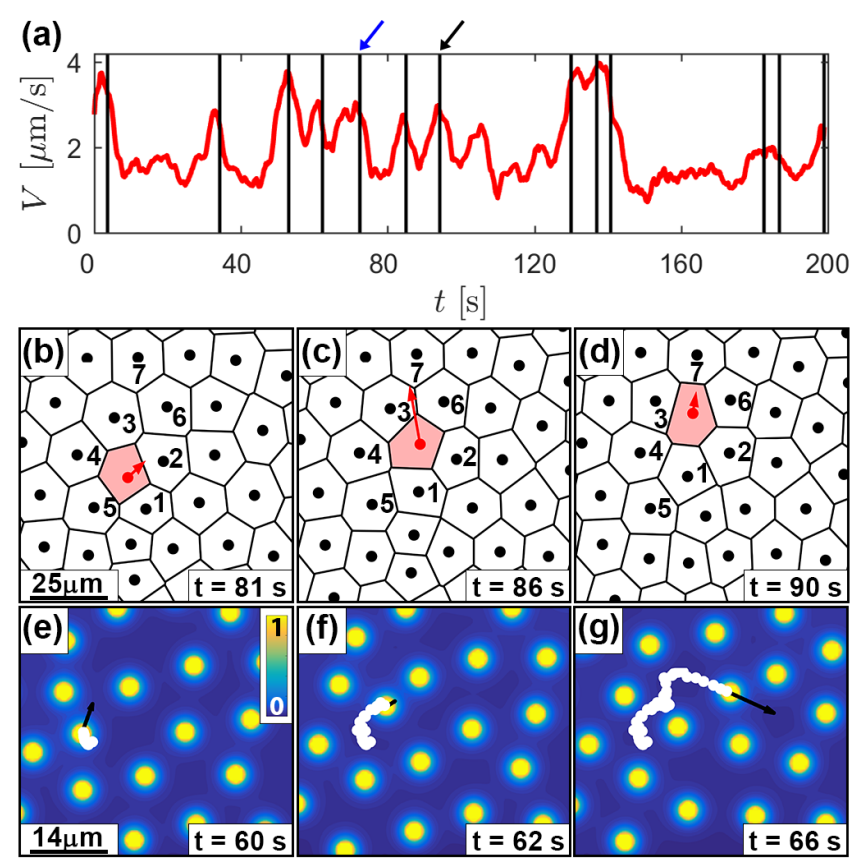

FIG. 3. Active atoms. (a) Time evolution of the velocity of an active atom in a crystalline monolayer with $L=10 R$ $\left(\left[\mathrm{H}_{2} \mathrm{O}_{2}\right]=3 \%, V_{0}=6 \mu \mathrm{m} / \mathrm{s}\right)$. The black vertical lines mark the times at which two (or more) nearest neighbors of the active particle change, suggesting a cell swap. (b)-(d) Corresponding Voronoi diagram (b) before, (c) during, and (d) after the cell swap indicated by the black arrow in (a). (e)-(g) Potential landscape and trajectory of the active atom (e),(f) before and (g) during the cell swap indicated by the blue arrow in (a). The potential is normalized by the value at the particle surface. In (b)-(g) the length of the arrows is proportional to the particle velocity.

Video S10 [23] ] when it points towards one of the Voronoi vertices, leading to a sudden cell swap [Fig. 3(d)]. A further description of the cell-swap mechanism is in [23]. At a first glance, this scenario can be compared to driving a colloidal particle at constant force through a 2D crystal [38], where the velocity is affected by its direction relative to the symmetry axes and by its local distance from the potential minima. However, there is one fundamental difference: active particles are capable of reorienting due to rotational diffusion, thus spontaneously sensing their environment. The dynamics is better understood by plotting the instantaneous local surrounding potential, which is depicted for another cell swap in Figs. 3(e)-3(g): in agreement with the Voronoi description, the swimming velocity shows maxima when the active atom self-propels through local potential minima [Fig. 3(f) and Video S11 [23] ].

Experimentally, while the two populations are clearly distinguishable [Figs. 1(g)-1(h)], active atoms show a broad range of coupling to lattice passive particles and we are unable to know a priori the exact value of $c$. The contact angle of Pt-coated particles confined at water-oil interfaces can in fact vary up to $20^{\circ}$ [22], which implies uncertainties on the coupling $c$ [23]. We, therefore, use
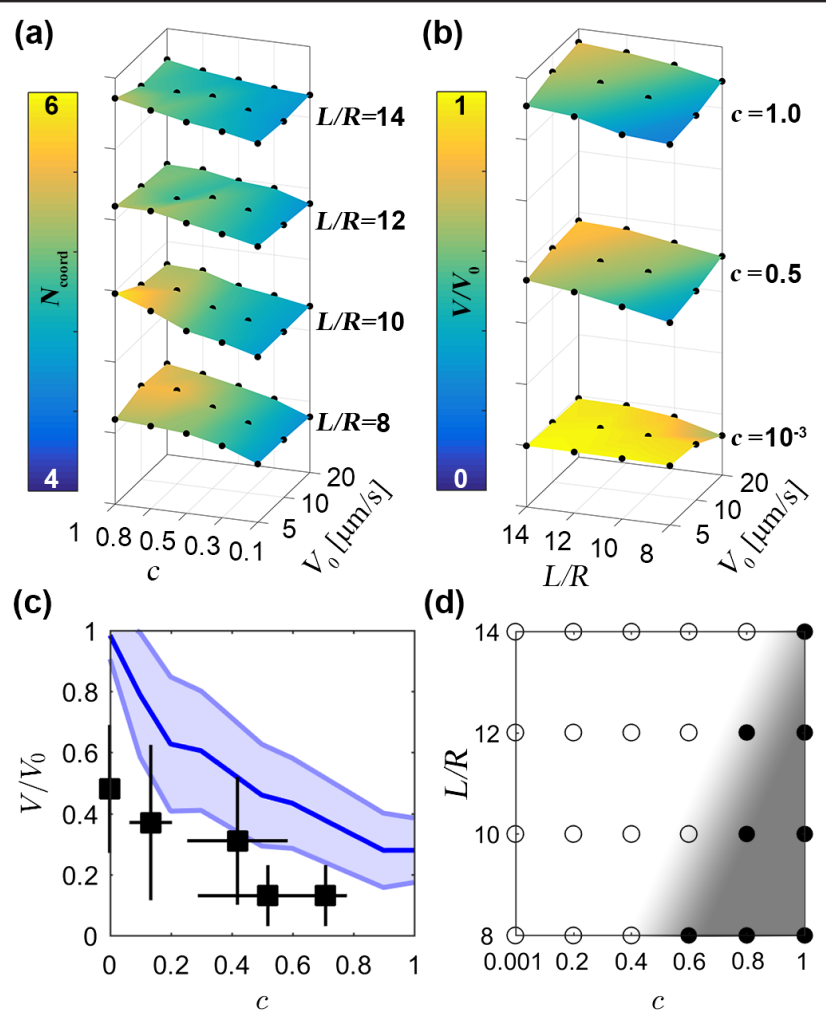

(d)

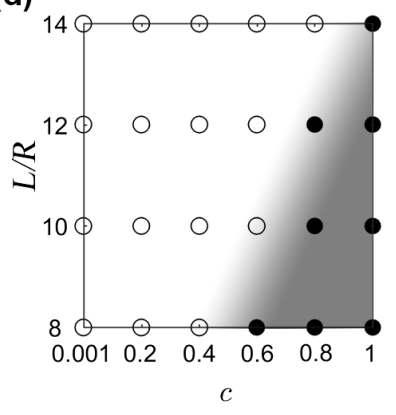

FIG. 4. (a),(b) Numerical color maps of (a) the coordination number $N_{\text {coord }}$ and (b) residual motility $V / V_{0}$, i.e., the mean velocity $V$ normalized by the mean free swimming speed $V_{0}$, as a function of $V_{0}, L$, and $c$. The dots correspond to the simulated values. (c) Experimental (black symbols) and numerical (blue line) mean velocity normalized by the maximum speed in the lattice $V_{\max }\left(\approx V_{0}\right)$, plotted as a function of the coupling $c$. The blue band marks the standard deviation of the numerical results. (d) Numerical data for fixed free swimming velocity $\left(V_{0}=1 \mu \mathrm{m} / \mathrm{s}\right)$ and variable $L$ and $c$, revealing mobile (empty symbols) and caged (filled symbols) states. A particle is labeled as caged if the maximum value of its root mean square displacement is smaller than $L$. The gray band highlights the transition.

numerical simulations to address the full dynamical response of the system as a function of the control parameters $L, V_{0}$, and $c$. We compute the mean coordination number $N_{\text {coord }}$ [Fig. 4(a)] and the mean residual motility $V / V_{0}$ [Fig. 4(b)] of active particles that self-propel through colloidal crystalline monolayers for $500 \mathrm{~s}$. The former reveals the extent to which self-propelling colloids belong to the hexagonal crystals and reaches its maximum value $\left(N_{\text {coord }} \approx 6\right)$ when highly coupled $(c \rightarrow 1)$ and slow $\left(V_{0} \rightarrow 0\right)$ particles roam in dense lattices [bottom stack in Fig. 4(a), the number of nearest neighbors sampled during the trajectories can be temporarily smaller or larger than six due to the variations in the local structure, leading to a weakly velocity-dependent $N_{\text {coord }}$ at $c=1$ ]. Larger swimming velocities locally destroy the hexagonal structure, whereas at lower couplings the active particles interact less with the lattice. The interplay between the active motion and the crystalline environments becomes weaker in sparser monolayers [top stack in Fig. 4(a)]. 
Similarly, a large loss of motility is reported in dense lattices and for high couplings [top stack in Fig. 4(b)], whereas active interstitials [bottom stack in Fig. 4(b), $c \rightarrow 0$ ] slow down only upon tumbling. A rough estimation of the experimental $c$ can be done by measuring the mean distance of the active particle from the nearest neighbor $\left\langle d_{\mathrm{NN}}\right\rangle$, at fixed $L$ and $V_{0}$ (see [23]). Figure 4(c) shows numerical (lines) and experimental (symbols) data of the residual motility for $L=10 R$ : in both instances, $V / V_{0}$ decreases with $c$ as a result of the stronger interactions with the crystalline landscape, although the experimental data are systematically smaller than the corresponding numerical simulations because interactions near contact and hydrodynamics are neglected in the calculations.

The results in Figs. 4(a)-4(c) are valid provided that the active particle can explore the surrounding environment. At small $V_{0}$ and large $c$ the active particle might be caged in one lattice position [solid circles in Fig. 4(d), Video S12 [23] ], while remaining free to move for smaller coupling values (empty circles), or larger $V_{0}$. Remarkably, an approximation of the swimming velocity required to avoid caging can be obtained by comparing Stokes' drag $F_{s} \sim 6 \pi \eta R V_{0}$ to the force needed to overcome the potential barrier $F_{p} \sim \nabla U / L$ [23].

In conclusion, we have investigated the motion of active particles in 2D loosely packed crystals at a fluid-fluid interface as a function of their coupling with the surrounding lattice. We identified two types of swimmers: active interstitials and active atoms. The different phenomenology depends on the orientation of the Janus colloids at the fluid interface, which in turn determines the magnitude of their electrostatic (dipolar) interactions, and can be observed at any fuel concentration, although for small $\left[\mathrm{H}_{2} \mathrm{O}_{2}\right]$ active atoms remain caged in their lattice positions. Hence, our results showcase the potential of fluid interfaces to realize structures where activity and microstructure can be decoupled. In particular, we envisage that active atoms can be used for the fabrication of new 2D active phases (e.g., active crystals and glasses).

We thank Paolo Malgaretti for discussions and ChiaoPeng Hsu for AFM measurements. K. D., I. B., and L. I. acknowledge financial support from the Swiss National Science Foundation Grant No. PP00P2 144646/1 and the ETH Zurich research Grants No. ETH-16 15-1 and No. FEL-02 14-1. I. B. acknowledges support from the European Commission with Marie Sklodowska-Curie individual Fellowship (Grant No. 747029). G. V. acknowledges funding by the European Commission with ERC Starting Grant ComplexSwimmers (Grant No. 677511).

*ivo.buttinoni@chem.ox.ac.uk

†lucio.isa@mat.ethz.ch

[1] J. R. Howse, R. A. L. Jones, A. J. Ryan, T. Gough, R. Vafabakhsh, and R. Golestanian, Phys. Rev. Lett. 99, 048102 (2007).
[2] H.-R. Jiang, N. Yoshinaga, and M. Sano, Phys. Rev. Lett. 105, 268302 (2010).

[3] J. Zhang, J. Yan, and S. Granick, Angew. Chem., Int. Ed. 55, 5166 (2016).

[4] C. Bechinger, R. Di Leonardo, H. Löwen, C. Reichhardt, G. Volpe, and G. Volpe, Rev. Mod. Phys. 88, 045006 (2016).

[5] D. Takagi, J. Palacci, A. B. Braunschweig, M. J. Shelley, and J. Zhang, Soft Matter 10, 1784 (2014).

[6] A. T. Brown, I. D. Vladescu, A. Dawson, T. Vissers, J. Schwarz-Linek, J.S. Lintuvuori, and W. C. Poon, Soft Matter 12, 131 (2016).

[7] S. Das, A. Garg, A. I. Campbell, J. Howse, A. Sen, D. Velegol, R. Golestanian, and S. J. Ebbens, Nat. Commun. 6, 8999 (2015).

[8] F. Ginot, I. Theurkauff, D. Levis, C. Ybert, L. Bocquet, L. Berthier, and C. Cottin-Bizonne, Phys. Rev. X 5, 011004 (2015).

[9] S. C. Takatori, R. De Dier, J. Vermant, and J. F. Brady, Nat. Commun. 7, 10694 (2016).

[10] F. Kümmel, P. Shabestari, C. Lozano, G. Volpe, and C. Bechinger, Soft Matter 11, 6187 (2015).

[11] J. Stenhammar, R. Wittkowski, D. Marenduzzo, and M. E. Cates, Phys. Rev. Lett. 114, 018301 (2015).

[12] B. van der Meer, L. Filion, and M. Dijkstra, Soft Matter 12, 3406 (2016).

[13] C. A. Weber, C. Bock, and E. Frey, Phys. Rev. Lett. 112, 168301 (2014).

[14] L. Berthier, Phys. Rev. Lett. 112, 220602 (2014).

[15] R. Mandal, P. J. Bhuyan, M. Rao, and C. Dasgupta, Soft Matter 12, 6268 (2016).

[16] I. Buttinoni, J. Bialké, F. Kümmel, H. Löwen, C. Bechinger, and T. Speck, Phys. Rev. Lett. 110, 238301 (2013).

[17] J. Palacci, S. Sacanna, A. P. Steinberg, D. J. Pine, and P. M. Chaikin, Science 339, 936 (2013).

[18] I. Theurkauff, C. Cottin-Bizonne, J. Palacci, C. Ybert, and L. Bocquet, Phys. Rev. Lett. 108, 268303 (2012).

[19] G. Briand and O. Dauchot, Phys. Rev. Lett. 117, 098004 (2016).

[20] P. Pieranski, Phys. Rev. Lett. 45, 569 (1980).

[21] B. J. Park, B. Lee, and T. Yu, Soft Matter 10, 9675 (2014).

[22] K. Dietrich, D. Renggli, M. Zanini, G. Volpe, I. Buttinoni, and L. Isa, New J. Phys. 19, 065008 (2017).

[23] See Supplemental Material at http://link.aps.org/ supplemental/10.1103/PhysRevLett.120.268004, which includes Refs. [24-29], for further details.

[24] J. Palacci, C. Cottin-Bizonne, C. Ybert, and L. Bocquet, Phys. Rev. Lett. 105, 088304 (2010).

[25] L. Baraban, M. Tasinkevych, M. N. Popescu, S. Sanchez, S. Dietrich, and O. Schmidt, Soft Matter 8, 48 (2012).

[26] A. I. Campbell and S. J. Ebbens, Langmuir 29, 14066 (2013).

[27] S. Ebbens, D. Gregory, G. Dunderdale, J. Howse, Y. Ibrahim, T. Liverpool, and R. Golestanian, Europhys. Lett. 106, 58003 (2014).

[28] M. Ohring, Materials Science of Thin Films (Academic Press, New York, 2001).

[29] P. A. Kralchevsky, K. D. Danov, and P. V. Petkov, Phil. Trans. R. Soc. A 374, 20150130 (2016).

[30] X. Wang, M. In, C. Blanc, M. Nobili, and A. Stocco, Soft Matter 11, 7376 (2015). 
[31] K. D. Danov and P. A. Kralchevsky, J. Colloid Interface Sci. 298, 213 (2006).

[32] G. Volpe, S. Gigan, and G. Volpe, Am. J. Phys. 82, 659 (2014).

[33] O. Chepizhko and F. Peruani, Phys. Rev. Lett. 111, 160604 (2013).

[34] X. Wang, M. In, C. Blanc, P. Malgaretti, M. Nobili, and A. Stocco, Faraday Discuss. 191, 305 (2016).
[35] K. D. Danov, P. A. Kralchevsky, B. N. Naydenov, and G. Brenn, J. Colloid Interface Sci. 287, 121 (2005).

[36] A. J. Hurd, J. Phys. A 18, L1055 (1985).

[37] M. Han, J. Yan, S. Granick, and E. Luijten, Proc. Natl. Acad. Sci. U.S.A. 114, 7513 (2017).

[38] B. Van Der Meer, W. Qi, R. G. Fokkink, J. Van Der Gucht, M. Dijkstra, and J. Sprakel, Proc. Natl. Acad. Sci. U.S.A. 111, 15356 (2014). 